\title{
DIVERSIDAD Y EDUCACIÓN ESPECIAL
}

\author{
Maritza Marín Cabrera ${ }^{1}$
}

\section{Resumen}

Este artículo sobre diversidad y educación especial, hace referencia a la importancia del respeto por la igualdad de oportunidades, los valores y actitudes en el ámbito educativo. Si bien es cierto, esta ha ido modificándose a lo largo de los años, aún queda mucho por mejorar, para llegar a que las escuelas sean inclusivas. Para que sea una realidad, debemos trabajar activamente.

Se toma en particular la participación de la persona con síndrome de Down integrados al Sistema Educativo Regular, quien con su esfuerzo e ilusiones participa de diversos programas de integración, y se convierte en ejemplo de tenacidad. A raíz del estudio, se visitaron varios centros de integración escolar en la etapa de preescolar y se percibieron necesidades de atención para la población en general. El artículo presenta varias estrategias metodológicas, con el fin de obtener un aprendizaje significativo y funcional, respetando la diversidad estudiantil. Por lo tanto, es un artículo de utilidad para las y los docentes, en especial, aquellos que laboran en la etapa de preescolar.

\section{Palabras claves}

Diversidad, igualdad de oportunidades, inclusión educativa, escuela para todos, derechos humanos, motivación, aprendizaje funcional.

\section{Abstract}

This article discusses diversity and special education, emphasizing the relevance of respect for equal opportunities as well as for values and attitudes within the school context. In spite of the fact that schools have improved in these aspects throughout the years, there is still lot of work to do in order to transform our schools in inclusive institutions.

Special attention is given to people with Down syndrome who are integrated to the regular system; students with dreams and illusions who participate in

1 Licenciada en Educación Especial con énfasis en Integración y Máster en Pedagegia con énfasis en Diversidad en los Procesos Educativos (por la UNA). Labora como profesora en la Escuela de Educación Especial Fernando Centeno Güiell. Académica de la División de Educación Básica desłe el año 2005. 
different integrated programs providing an example of tenacity. Furthermore, several pre-school integration centers were visited and a lot of necessities of attention to the general population were detected. This article presents different methodological strategies designed to produce meaningful and functional learning processes, respecting student's di- versity. Accordingly, this is a useful article to educators, especially those who work with pre-schooled children.

\section{Key Words}

Diversity, special education, equal opportunities, inclusive education, Down syndrome, pre-school classrooms, teachers

\section{L}

os estudios y prácticas que da la educación para la diversidad, se han gela complejidad de este proceso desde una visión transdiciplinar y holística, que implica contemplar las diferentes aristas de una realidad latente en nuestras sociedades e involucra un pensamiento más reflexivo, crítico, que genere una atención sostenida y permanente a las diferencias. Se quiere sobrepasar las barreras de una atención asistencialista, en función de las dificultades de los y las estudiantes que no aprenden.

Cabe destacar que aún hoy, la diversidad humana, en el contexto educacional, sigue viéndose de manera restringida, y se concibe como un problema que dificulta los procesos de enseñanza y aprendizaje; no como una característica propia e integrada en la experiencia cotidiana de las personas. De acuerdo con lo anterior, en educación los términos de atención a la diversidad y educación especial, se han interpretado de manera simplista, y se dejan de lado aspectos sobresalientes de la teoría y la práctica en estas áreas.

El concepto de diversidad no se puede ni debe pensar en términos restringidos. Como lo expresa Melero (citado por Sánchez y Torres, 2002, p. 64), "educar en la diversidad no se basa en la adopción de medidas excepcionales para las personas con necesidades educativas específicas, sino en la adopción de un modelo de currículum que facilite el aprendizaje de todos los alumnos y alumnas en su diversidad".

La visión restringida, según este autor, debe superar la equivocación que existe en el pensamiento de los profesionales, al considerar los programas de integración escolar como una reforma de la educación especial y no de la educación general. La atención a la diversidad, es un principio que se refiere a una pluralidad de identidades y condición de la existencia humana. Actualmente, Costa Rica se encuentra en un momento de transición que tiende a crear las condiciones para que las y'los estudiantes puedan participar en 
el ámbito educativo en igualdad de oportunidades. De esta manera, se promueve una inclusión educativa que, según Meléndez (2002), es:

"La potencialización de las diversas perspectivas de mundo aprovechándose de la convivencia solidaria de los aprendizajes, donde la exclusión y la discriminación no tienen cabida; sino que, más bien, las oportunidades de desarrollo se construyen con la participación equitativa de todos los miembros de una comunidad escolar inclusiva en la que todas las identidades valen".

Se pretende pasar de creer que la educación especial es una modalidad educativa independiente y separada del sistema educativo regular, a considerarla como una parte integrante de este, como un conjunto de medios y recursos, humanos y materiales, puestos al servicio del sistema educativo, para la atención de las necesidades educativas especiales que pueda presentar la o el estudiante a lo largo de su escolaridad.

Como respuesta a la diversidad en el ámbito educativo y lucha contra la exclusión de las personas, aparece una nueva corriente que tuvo su origen en la Conferencia Mundial de Educación para Todos (Tailandia, 1990), que busca la implementación de escuela para todos. Según la UNESCO (1994), tiene como función ser capaz de dar una educación de calidad a todos los niños y niñas; con su planteamiento se da un paso muy importante, al intentar cambiar las actitudes de discriminación y crear comunidades que acojan las diferencias en el marco de socicdades integradoras. De lo anterior se deriva que la denominada "escuela para todos" no es para un tipo de niños y niñas en especial, sino la que se propone educar a todos y todas, poniendo en práctica el principio de atención a la diversidad.

En definitiva, la escuela para todos, al asumir el derecho a la educación en su sentido más pleno e integrador, pretende promover un cambio no sólo en el currículo y la enseñanza, sino también, en la organización de los centros, de forma que la escuela se convierta en un eslabón más hacia la autonomía e independencia de todas las personas, en sociedades pluralistas.

La UNESCO (1994) indica que existe la necesidad de cambiar la perspectiva social. Durante mucho tiempo, las dificultades de las personas con discapacidad han sido agravadas por una sociedad que invalida y ñja más la atención en su discapacidad que en su potencial. Por un lado, aún no está del todo superado el hecho de que el fracaso escolar se le atribuye solamente al estudiante, debido a sus deficiencias; pero, por otro, ya se empieza a 
cuestionar cada vez más el papel de la escuela y hasta qué punto es esta -con sus metodologías, estrategias, currículo- la que falla, al no ser capaz de dar respuesta a las particularidades de los y las estudiantes. De esta manera, la escuela para todos busca una reforma estructural, cuyas propuestas se concreten en la denominada escuela inclusiva.

Los argumentos que demandan la reforma de la educación especial se fundamentan en algunos datos y hechos reales y en los análisis críticos de la situación actual, los cuales, según Lipsky y Gartner (citados por Vega, 2001), son:

- Los alumnos de educación especial obtienen resultados escasos. La tasa de desempleo entre las personas con discapacidad es la más alta de todos los grupos de la población.

- Las organizaciones de padres insisten en la necesidad de la escuela inclusiva.

- La educación inclusiva es un derecho de todas las personas. Afirman que si las personas con discapacidad han estado excluidas, difícilmente pueden ser aceptadas como personas con derechos (p. 50).

Las escuelas deberían constituir entornos de aprendizaje para sus estudiantes y cuestionar el contenido y el contexto, así como las disciplinas tradicionales, las agrupaciones y cualquier actividad que demuestre segregación, discriminación o exclusión.

Melero (citado por Sánchez y Torres, 2002) indica que el docente ha de sentirse comprometido con la construcción de una sociedad más justa, equitativa y solidaria y ser consecuente con este compromiso fuera y dentro del aula: el planteamiento de la atención a la diversidad no se centra sólo en las características individuales, sino en la interacción de estas con las condiciones de enseñanza y aprendizaje que proporciona la escuela, el origen familiar, cultural y social.

En este sentido, a medida que la experiencia docente y la investigación aporten nuevos conocimientos sobre la atención a la diversidad, también se tendrá mayor capacidad de ofrecer respuestas acordes con las necesidades personales y sociales. El valor de esa diversidad es la que brindará a los seres humanos la calidad que se merecen. 


\section{De la integración a la inclusión educativa}

La educación inclusiva, con frecuencia, se asocia con la participación de estudiantes con discapacidad en las escuelas del sistema educativo regular; es decir, se utiliza el término de inclusión como sinónimo de integración; no obstante, se trata de dos conceptos distintos, aunque relacionados entre sí.

La integración educativa ha constituido un movimiento importante para promover el acceso y la participación de las personas con discapacidad en aulas regulares. Mientras que la inclusión educativa es un concepto más amplio, ya que no se trata sólo de lograr acceso a la escuela regular de determinado grupo de estudiantes que han sido excluidos, sino también, de transformar el sistema educativo en su conjunto para atender la diversidad de necesidades educativas de las personas, y asegurar la igualdad de oportunidades en el aprendizaje y su plena participación e integración.

La educación inclusiva implica la eliminación de las barreras que existen para el aprendizaje, con el objetivo principal de que las diferencias culturales, socioeconómicas, individuales y de género, no se conviertan en desigualdades educativas.

El movimiento de la educación inclusiva aspira a hacer efectivo, para todos, el derecho a la educación, a la igualdad de oportunidades y a la participación; la escuela es la que debe adaptarse a los niños y niñas y no estos a la escuela.

Fullan (UNESCO, 1994) indica, en la conferencia sobre Necesidades Educativas Especiales: Acceso y Calidad, que:

"Para reformar la educación especial, es necesario tener en cuenta todos los aspectos que se consideran en general en una reforma de la educación. La inclusión no se logra con facilidad. Es el resultado de un complejo proceso de integración de cambios cualitativos y cuantitativos, necesarios para definir y aplicar las soluciones adecuadas. Para lograr el cambio, se precisan constancia, coordinación, seguimiento, resolución de conflictos, etc., es decir una buena conducción a todos los niveles" (p. 39).

De lo anterior se desprende que, para lograr avanzar en los programas de inclusión, es necesario el esfuerzo colectivo de la sociedad en su conjunto, que es el principio de la educación responsabilidad de todos y todas. 
La inclusión es beneficiosa para cada estudiante, ya que aprende con una metodología más individualizada, dispone de mayores recursos y desarrolla valores y actitudes de solidaridad, respeto y colaboración. Implica que se produzcan cambios en el currículo, la metodología y la organización de las escuelas, así como en la modificación de las condiciones que dejan fuera a niños y niñas con neceșidades educativas especiales.

Stainback y Stainback (1999) proponen tres razones a favor de la inclusión e indican que esta debe servir:

"Para ofrecer a cada estudiante la posibilidad de aprender a vivir y trabajar con sus iguales en contextos naturales, de educación integrada y comunidad; para evitar los efectos inherentes a la segregación cuando los èstudiantes están en lugares separados, en aulas o centros de educación especial, y para hacer lo que es justo y equitativo" (p. 50).

Costa Rica se encuentra en un proceso de transición, en el que se da paso de la segregación y exclusión a los procesos de integración escolar y social de las personas con discapacidad. Se sigue con esta lucha y se incluyen el respeto hacia la diversidad humana y la igualdad de oportunidades; por lo tanto, a la inclusión en la atención educativa de todas las personas.

A pesar de existir, mundialmente, un cambio de paradigma en la atención de las personas con necesidades educativas especiales, la comprensión, interpretación y compromiso tanto individual como social, llevan a un proceso complejo, que combina concepciones arraigadas a paradigmas anteriores, resistencias al cambio y hasta ajustes económicos y estructurales de las instituciones.

A pesar de reconocer la importancia del derecho a la educación de cada persona, la respuesta que se da a quienes tienen una discapacidad es aún marginal y segregadora.

En este sentido, el Foro Mundial sobre Educación (Dakar, 2000) da énfasis a las estrategias metodológicas que garanticen la atención de las necesidades básicas del aprendizaje de todos los niños y las niñas. Algunos de lós cambios experimentados, que han permitido la modificación de conceptos como la integración escolar, son la implementación de programas como "una educación para todos", en la que el principio fundamental, es que los niños y las niñas deben aprender juntos. 


\section{Inclusión escolar y síndrome de Down}

Durante los últimos años, se ha evidenciado en el Sistema Educativo de Costa Rica un incremento en la atención de estudiantes con necesidades de apoyos educativos variados e importantes. La atención de la persona con discapacidad ha sido un reto para las sociedades en general; razón por la cual han hecho su aparición a lo largo de la historia (dependiendo de las creencias y los valores de cada momento), diferentes enfoques que han intentado dar respuesta a la forma de abordarla, desde considerarlos como manifestación del mal, objetos de rehabilitación, hasta la lucha por la integración e inclusión social y escolar en igualdad de condiciones. Poco a poco, nuestra sociedad asume que la humanidad se enriquece con la diversidad y que tener discapacidad es una manera más de ser y de estar en este mundo de todos.

Actualmente, gran cantidad de personas con síndrome de Down se han integrado al sistema educativo regular. Un ejemplo de lo anterior, es el Departamento de Retardo Mental del Centro Nacional de Educación Especial Fernando Centeno Güell, el cual consideró que para iniciar los procesos de integración, era valioso contar con el apoyo pedagógico de docentes que tenían a su cargo la atención de los niños y niñas con síndrome de Down. Se dio a la tarea de sistematizar las experiencias vividas en el programa de integración en coordinación con otros profesionales, que tenían a su cargo el trabajo del área social, psicología y de enfermería. Es entonces, cuando en el año 1997, se integran en el sistema educativo regular a 5 niños y 2 niñas con síndrome de Down, que fueron un enlace entre la educación especial y la educación regular. Un trabajo en equipo puede ayudar significativamente al logro de objetivos, metas de los y las estudiantes.

Como dato significativo, durante el 2006, la Escuela Fernando Centeno Güell atiende en su programa a 25 niños con síndrome de Down integrados al Sistema Educativo Regular. Por lo tanto, la cantidad de niños y niñas integrados aumentó significativamente entre 1997 y el 2006.

Se tuvo la oportunidad, en el año 2004, de realizar entrevistas y observaciones a docentes de la Etapa de Transición del Sistema Educativo Regular, quienes tienen en sus aulas diversidad estudiantil, incluyendo un niño o una niña con síndrome de Down. Se destaca que muestran dudas, tienen interrogantes e incertidumbre sobre la atención idónea de las diferencias individuales. Por lo que, al pensar en una escuela que labore para la atención de la diversidad, se debe poner atención a la importancia del rol docente para el 
éxito del reto planteado y, por lo tanto, al apoyo, la sensibilización, el trabajo en equipo y la capacitación que se les pueda brindar.

Aguilar (2000, p. 113) plantea que "sin un profesional preparado y responsable, difícilmente podrán tener éxito los cambios esperados, ni aplicarse cualquier programa más o menos innovador". Los planteamientos de una escuela para la diversidad, implican un cambio en el concepto mismo de educación.

Mehroto (citado por MEP, 2000, p. 13), indica que "los programas integrales dirigidos a la primera infancia deben contribuir con la equidad social y eliminar discriminaciones entre niños y niñas: asegurar la participación de ambos teniendo en cuenta sus potencialidades más que sus diferencias". Según lo anterior, la educación preescolar debe ser orientada al desarrollo de la personalidad y las capacidades del niño y la niña, e inculcarles el respeto de los derechos humanos y el valor de la individualidad.

A continuación, se proponen estrategias curriculares, que se adaptan y responden de manera idónea, a la realidad y a las necesidades que se plantean cotidianamente en las escuelas, para que las y los estudiantes desarrollen al máximo sus capacidades, de tal modo que las diferencias no se conviertan en desigualdades; se toma en cuenta, la importancia de establecer variedad y diversidad de actividades que se basen en las potencialidades, las necesidades y los ritmos de aprendizaje de cada estudiante. El interés radica en que, en los primeros años de vida del estudiante, se construyen las bases de su autoestima, desarrolla confianza, seguridad, autonomía e iniciativa, en relación con otras personas y con la cultura a la que pertenece. Un docente capacitado para abordar la diversidad de estudiantes, es fundamental para lograr éxito en los siguientes niveles de escolarización.

Las y los docentes que atienden en sus aulas a diversidad estudiantil, incluyendo síndrome de Down, tienen algunas necesidades en su labor diaria. Por eso, es importante diseñar estrategias curriculares para brindar igualdad de oportunidades en sus salones de clase. Algunas de ellas son:

- Potenciar la máxima heterogeneidad entre los y las estudiantes a la hora de agruparlos.

- Potenciar la interdependencia positiva, de manera que al organizar las experiencias de aprendizaje, se consiga que los niños y las niñas comprendan que se necesitan unos a otros para conseguir los objetivos.

- Potenciar los logros individuales, combinando el aprendizaje en grupos cooperativos y el aprendizaje individual para que cada niño y niña, 
pueda desarrollar sus habilidades personales, tomando como base su potencial y ritmo de aprendizaje. Esto se logra diversificando las metas personales, favoreciendo la autonomía, la toma de decisiones y fomentando la autoevaluación.

Proporcionar materiales apropiados y sugerencias de cómo realizar un trabajo. En el caso, las y los estudiantes con discapacidad, en ocasiones, requieren de adaptación de materiales acordes con sus potencialidades y necesidades.

Observar la interacción entre las y los estudiantes, con el fin de conocer qué problemas encuentran para trabajar en forma grupal.

Utilizar las agrupaciones espontáneas, para mejorar las relaciones entre los y las estudiantes, tanto fuera como dentro del aula.

Respetar la diversidad cultural.

Respetar la diversidad de religiones que tienen las familias de nuestros estudiantes y ellos mismos.

Modelar los valores referentes a la igualdad de oportunidades

Evitar la clasificación entre los estudiantes, como, por ejemplo, dar la indicación de realizar las filas de acuerdo con el género, mujeres y hombres y por orden de tamaño.

Conocer la situación de partida de las y los estudiantes, incluidos sus conocimientos previos, y sobre todo de su estilo de aprendizaje, para proveer estrategias didácticas adaptadas a sus habilidades y necesidades.

Mostrar igualdad en cuanto a las responsabilidades de las y los estudiantes con la limpieza del aula.

Cada niño y cada niña tienen que percibir que consiguen algo de éxito en la tarea, ya que esto les va a permitir obtener una imagen positiva del aprendizaje.

Garantizar la movilidad de las y los estudiantes, sin que sus desplazamientos interfieran en el trabajo del resto del grupo.

Organizar el espacio, pues puede facilitar o dificultar la atención de niños y niñas con dificultades motoras o sensoriales. El uso común de los materiales y su accesibilidad, son ejemplos de organización del espacio a tomar en cuenta para atender la diversidad en este aspecto.

Fomentar la diversiỏad también en las śreas de rincones; un ejemplo de ello es el rincón de cívica, el cual debe contar con elementos de diversos países, según sea el origen de niños y niñas. Además, si los materiales están asequibies, despertarán en las y los estudiantes la curiosidad y 
el interés necesarios para que las diferentes propuestas que se ofrecen se aprovechen al máximo. Esta elección favorece el que ningún niño o niña sienta una situación de fracaso, ya que cada cual puede actuar basándose en sus posibilidades y limitaciones y todos pueden seguir trayectorias diferentes para lograr aprendizajes.

- Potenciar, mediante la organización de los rincones, todos los aspectos del desarrollo de los niños y las niñas.

- Mostrar igualdad de oportunidades en cuanto a género, al decorar las paredes del aula. Por ejemplo, murales de niñas y niños en el área de casita y al igual que en el área de construcción.

- Los procesos de aprendizaje basados en los intereses de las y los estudiantes y el tratamiento globalizador del currículum, deben ser la base del principio de flexibilidad.

- Fomentar la intervención educativa en diversas estrategias didácticas. Seleccionar materiales que, siendo indicados para el alumno o la alumna con discapacidad, resulten valiosos para los demás.

- Seleccionar materiales que refuercen las habilidades e intereses de los alumnos y las alumnas.

- Ubicar el material didáctico de manera accesible para los niños y las niñas, sin que tengan que recurrir al adulto para tomar aquellos que necesiten y, por lo tanto, potenciar la autonomía y la libre elección.

- Elaborar unidades didácticas interesantes para los niños y las niñas, con la intención de potenciar su implicación física, cognitiva, afectiva y social.

- $\quad$ Propiciar tareas y actividades significativas para que se pueda producir un aprendizaje funcional en los niños y las niñas.

- Las actividades deben diversificarse según el grado de dificultad del estudiante, de menor a mayor, de forma que todos los alumnos y las alumnas estén realizando alguna tarea adecuada a sus capacidades. Las actividades tienen que proporcionar situaciones para que todos puedan sentirse competentes y alcanzar éxito en la tarea.

- Partir de la motivación e interés de las y los estudiantes. La motivación es un requisito indispensable para que se produzca el aprendizaje. Esto requiere tanto conocer la situación familiar y personal del alumno y la alumna, como la percepción y la imagen que se esté construyendo de sí mismo. 
- Dejar de lado los métodos de evaluación rígidos y poco significativos para los y las estudiantes. La autoevaluación desde edades tempranas, es una manera de hacer estudiantes críticos y creando sistemas educativos que reflejen la calidad de la educación, en una escuela para todos.

- La evaluación continua es el instrumento potenciador del tratamiento diversificado de los obstáculos, dificultades o logros de cada uno. Fomentar en la evaluación técnicas de observación.

- Dar importancia al currículo oculto, especialmente, en lo que respecta al ámbito de la educación de valores y actitudes.

Como conclusión, las alumnas y los alumnos trabajan y aprenden si se aceptan las diferencias existentes entre ellos y se ajustan en consecuencia los objetivos, los contenidos, las actividades de enseñanza y aprendizaje y las actividades de evaluación. Los estudiantes más motivados, aprenden cuando comprueban que tienen éxito en la realización de las tareas, sintiéndose, en este caso, más competentes y mejorando su autoestima.

A todas las personas con síndrome de Down, quienes mantienen viva la luz de la esperanza con su esfuerzo e ilusiones y a todos y todas los que creen en la diversidad estudiantil, les insto a seguir adelante en la lucha por el respeto, por la calidad de vida y la igualdad de oportunidades en beneficio de la humanidad. 


\section{REFERENCIAS}

Aguilar, L. (2000) De la integración a la inclusividad. Buenos Aires, Argentina. Editorial Espacio.

Agüero, R. y otros. (2003) Integración del Niño con Síndrome de Down en el Sistema Educativo Regular: La experiencia de un grupo de Padres de Familia del Centro Nacional de Educación Especial Fernando Centeno Güell. Universidad Nacional. Heredia. Costa Rica.

Asamblea Legislativa (1996). Ley 7600. Igualdad de Oportunidades para las personas con discapacidad en Costa Rica. San José, Costa Rica: Editorial Imprenta Nacional.

López, N. y Lou, M. (1998) Bases Psicopedagógicas de la Educación Especial. Madrid, España: Editorial Pirámide.

Meléndez, L. (2002) La inclusión escolar del alumno con discapacidad intelectual. Bogotá, Colombia: Editorial GLARP.

Ministerio de Educación Pública. (2000) Programa de estudio del Ciclo Materno Infantil. San José, Costa Rica: Editorial Ministerio.

Stainback, S. y Stainback, W. (1999) Aulas Inclusivas. Madrid, España: Editorial Narcea.

UNESCO. (1991) La integración de niños discapacitados a la educación común. Santiago, Chile.

UNESCO. (1994) Conferencia Mundial sobre Necesidades Educativas Especiales, Acceso y Calidad. Declaración de Salamanca y Marco de Acción sobre Necesidades Educativas Especiales. Salamanca, España: Ministerio de Educación y Ciencia.

UNESCO. (2000) Marco de Acción de Dakar. Educación para Todos. Dakar, África. Vega, A. (2001) La educación de los niños con Síndrome de Down. Principios y prácticas. Salamanca, España: Editorial Amarú. 